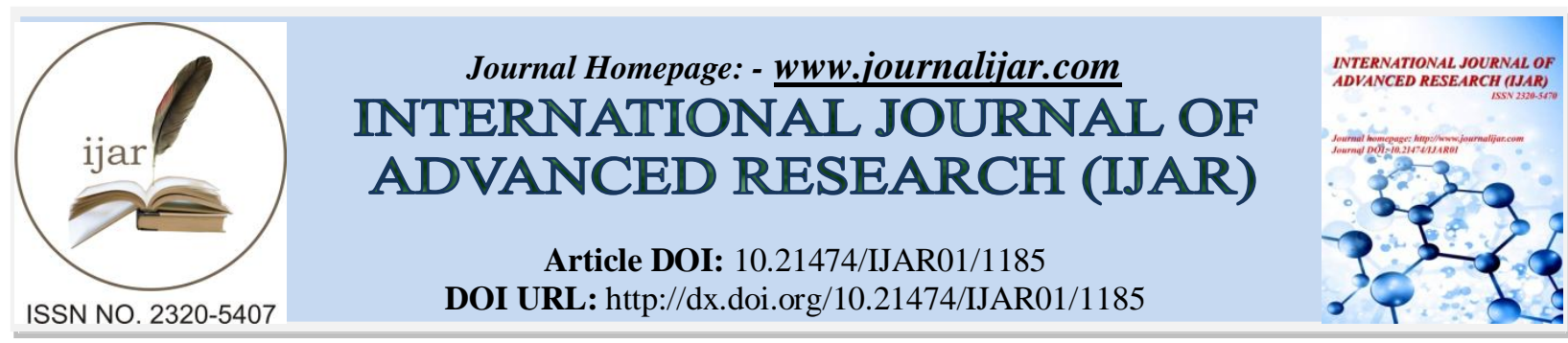

RESEARCH ARTICLE

\title{
MORPHOMETRIC AND MERISTIC CHARACTERS OF MULLUS SERMULETUS (LINNEAUS, 1758) AND MULLUS BARBATUS (LINNEAUS, 1758) FROM MEDITERRANEAN SEA, ALEXANDRIA, EGYPT.
}

Usama M. Mahmoud ${ }^{1}$, Fahmy I. El-Gamal ${ }^{2}$, Sahar F. Mehanna ${ }^{2}$ and "Eman Mansour².

1. Department of Zoology, Faculty of Science, Assiut University, Assiut, Egypt.

2. National Institute of Oceanography and Fisheries, Egypt.

\section{Manuscript Info}

Manuscript History

Received: 19 June 2016

Final Accepted: 16 July 2016

Published: August 2016

Key words:-

Morhpometric, Meristic, Mullus sermuletus, Mullus barbatus,

Alexandria, Egypt..

\section{Abstract}

In the present investigation, Intra-and inter-specific variations in morphometric indices of Mullus sermuletus and Mullus barbatus, from Mediterranean Sea at Alexandria, Egypt, were revealed. The type of allometry of morphometric measurements showed that for both species studied, females showed isometric measurements more than males and M. sermuletus showed isometric measurements more than $M$. barbatus for combined sexes. Meristic characters are significantly different between the two species studied. No sexual dimorphism was found in the meristic characters of the two species under study.

Copy Right, IJAR, 2016,. All rights reserved.

\section{Introduction:-}

Morphometric characters of fishes were found to be of taxonomic importance in sex, race and species identification by many investigators (Nelson, 1984; whitehead et al., 1986; Golani, 1994; Mekkawy, 1987\&1994; Mahmoud, 1988, 1991\& 1993; Harabawy, 1993\& 2002; Khalil et al., 1983\& 1984; Oliveira and Almada, 1995; Osman, 2000; Costa et al., 2003; Obady, 2003; Smith and Paulin, 2003; Basmidi, 2004; Turan, 2004; Randall and Heemstra, 2009; Randall and King, 2009; Lawson, 2010; Simon et al., 2010; Elamin et al., 2011; Mekkawy and Mohammad, 2011; Mazlan et al., 2012; Deepti et al., 2013; Sajina et al., 2013; Uiblein and Heemstra, 2010 \&2011; Abbaspour et al., 2013; Safi et al., 2014; Jawad, 2015; Masood et al., 2015; Zubia et al., 2015 and Mahmoud et al., $2016 \mathrm{a}, \mathrm{b})$.

The meristic characters were also found to be valid in sex, race and species identification (Mahmoud and Mekkawy, 1991; Mahmoud, 1991, 1993\&2002; Mekkawy, 1991\& 1997; Costa et al., 2003; Obady, 2003; Basmidi, 2004; Turan, 2004; Randall and Heemstra, 2009; Randall and King, 2009; Lawson, 2010; Simon et al., 2010; Elamin et al., 2011; Mekkawy and Mohammad, 2011; Uiblein and Heemstra, 2010 \& 2011; Safi et al., 2014; Jawad, 2015; Masood et al., 2015; Zubia et al., 2015 and Mahmoud et al., 2016 a, b) .

In the present work, the morphometric and meristic characters were used to reveal intra- and inter-specific variations of stripped red mullet (Mullus surmuletus, Linnaeus, 1758) and red mullet (Mullus barbatus, Linnaeus, 1758) from Mediterranean sea at Alexanderia, Egypt.

Corresponding Author:- Eman Mansour.

Address:- National Institute of Oceanography and Fisheries, Egypt. 


\section{Matreials and Methods:-}

In the present study, 105 males (78-165 mm in Standard Length "SL" ) and 123 females (82-187 mm in SL ) of Mullus sermuletus and 100 males (85-138 mm in SL) and 107 females (90- $153 \mathrm{~mm}$ in SL ) of Mullus barbatus were randomly collected from Mediterranean sea at Alexanderia, Egypt during the period from January 2015 to December 2015.

\section{Morphometrics:-}

For each fish, 22 morphometric measurements were made on the left side up to nearest millimeter using divider and measuring board. The following is a list of these measurements which are represented in figure (1); each measurement is labeled on this figure by its corresponding number indicated in such a list. Those morphometric measurements included:

1. Total length (TL).

2. Forked length (FL).

3. Standard length (SL).

4. Pre- $1^{\text {st }}$ Dorsal length (PrD1L).

5. Pre-Pectoral length (PrPL).

6. Pre-Ventral length (PrVL).

7. Pre-Anal length (PrAL).

8. Head length (HL).

9. Pre-Orbital length (PrOL).

10. Eye diameter (ED).

11. Post-Orbital length (PtOL).

12. Inter-Orbital width (IOW).

13. Body depth at anus (BD).

14. Caudal peduncle depth (CPD).

15. Distance between $1^{\text {st }}$ dorsal fin and ventral fin origins (D1VOFL).

16. Distance between $2^{\text {nd }}$ dorsal and anal fin ends (D2AEFL).

17. Distance between $1^{\text {st }}$ dorsal fin origin and the end of anal fin (D1OAEFL).

18. Distance between $2^{\text {nd }}$ dorsal fin end and ventral fin origin (D2EVOFL).

19. Distance between $2^{\text {nd }}$ dorsal fin end and ventral caudal fin origin (D2EvCFL).

20. Distance between $2^{\text {nd }}$ dorsal fin end and dorsal caudal fin origin (D2EdCFL).

21. Distance between anal fin end and ventral caudal fin origin (AEvCFL).

22. Distance between anal fin end and dorsal caudal fin origin (AEdCFL).

\section{Meristics:-}

The following meristic counts were recorded:

1. Number of the pectoral fin rays (PFR).

2. Number of gill rakers on the epibranchial portion of the first left gill arch (ascending) (UGR).

3. Number of gill rakers on ceratohypobranchial portion of the first left gill arch (descending) (LGR).

4. Total number of gill rakers on the first left gill arch (TGR). 


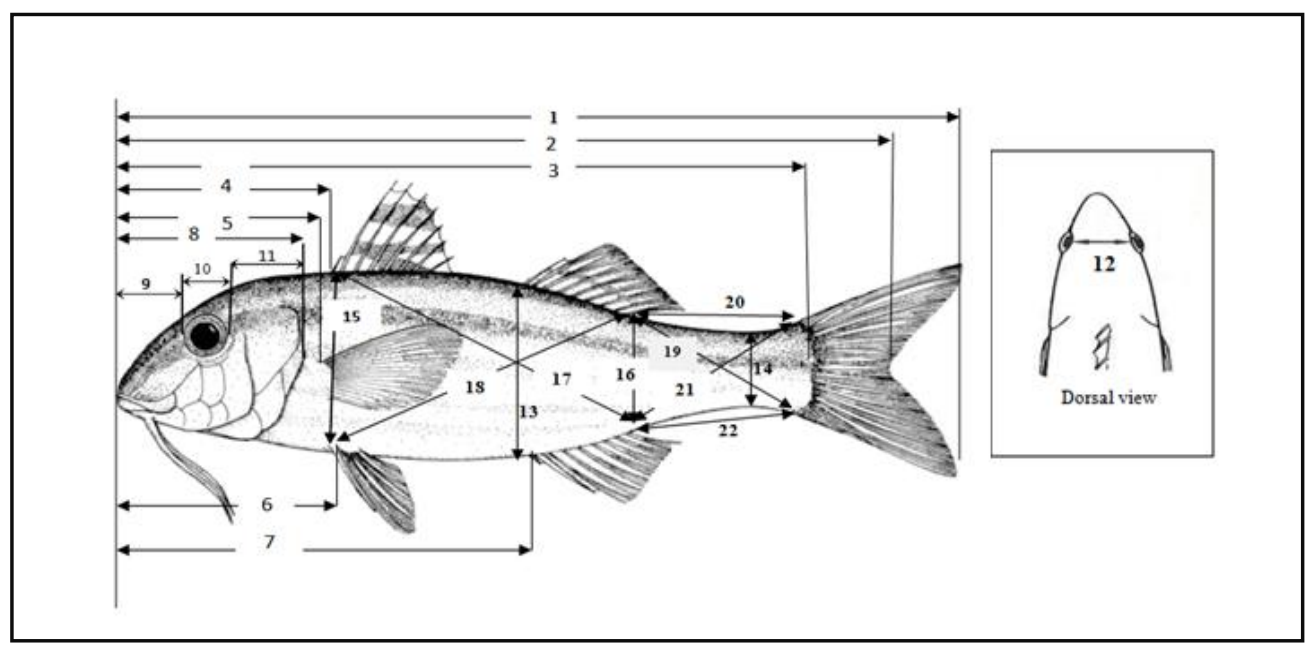

Fig. 1:- Schematic illustration of measurements taken on the body of the two Mullidae species from Mediterranean Sea at Alexandria, Egypt.

\section{Statistical analysis:-}

The basic statistics (mean, standard error and range) of certain morphometric indices (relative to standard length, SL or head length, HL) and meristic characters were estimated. The allometric coefficients of the raw morphometric characters and their relationships with fish size (SL) were estimated using power function equation and the linear regression model respectively. The simple power function or allometry equation of Huxely (1932):

$$
\mathrm{Y}=\mathrm{a} \mathrm{X}^{\mathrm{b}}
$$

was used, where $\mathrm{Y}$ and $\mathrm{X}$ are dependent and independent variables respectively and $\mathrm{a}$ and $\mathrm{b}$ (the allometric coefficient) are constants. The parameters $\mathrm{a}$ and $\mathrm{b}$ of this equation were estimated by fitting a linear equation to the logarithmic values of $\mathrm{Y}$ and $\mathrm{X}$ according to the least square method. This leads to an equation of the form:

$\log Y=\log a+b \log X$

Moreover, the type of allometry was determined by estimating of confidence limits of the allometric coefficients, isometry (I), negative allometry (-) or positive allometry (+). The type of allometry was evaluated by testifying the significance of the allometric coefficients $(b)(b=1, b>1$, and $b<1$ for isometric growth, positive allometric growth and negative allometric growth respectively) that serves as a criterion for the intensity of differential increase in the morphological measurements relative to a certain reference length. The type of allometry was found to be helpful for studying intra-and inter-specific variation of Mullus sermuletus and Mullus barbatus. The mean values of meristic characters within and between species considered were testified by t-test.

Statistical analyses for morphometric and meristic data were performed using the SPSS version 18 software package and Excel (Microsoft office, 2007).

\section{Results:-}

Morphometrics:-

The relationship between the morphometric measurements and fish size (SL) of Mullus sermuletus and Mullus barbatus were best described by the linear regression equations (Tables $1 \& 2$ ).

The basic statistics of the morphometric indices (relative to SL or HL) of M. sermuletus considered show sexual dimorphism (Table 3\&4). IOW/SL, BD/SL, D1VOFL/SL, D2AEFL/SL, D2EdCFl/SL, PrD1L/HL, PrPL/HL and PrOL/HL are indices to be size-free and so valid as discriminating tool between males and females of $M$. sermuletus.

The basic statistics of the morphometric indices (relative to SL or HL) of M. barbatus considered show sexual dimorphism (Table 5\&6). PrAL/SL, PrOL/SL, ED/SL, BD/SL, CPD/SL, D2AEFL/SL, AEdCFL/SL, CPD/HL, D2AEFL/HL and D1OAEFL/HL are indices to be size free and so valid as discriminating tool between males and females of $M$. barbatus. 
The basic statistics of the morphometric indices (relative to SL or HL) of $M$. sermuletus and M. barbatus considered show inter-specific variations (Tables 7\&8). All morphometric indices are significantly different except for PtOL/SL, D1OAEFL/SL, D2EVOFL/SL, D2EvCFL/SL, D2EdCFL/SL and IOW/HL.

The patterns of variations in the morphometric measurements of $M$. sermuletus and M. barbatus were considered in terms of their mode of growth (i.e, their type of allometry). For both M. sermuletus and M. barbatus, females show isometric characters more than males while the morphometric measurements of combined sexes of $M$. sermuletus show isometric growth more than M. barbatus (Tables $9 \& 10$ )

\section{Meristics:-}

The pectoral fin rays and upper, lower and total gill raker counts of males and females and combined sexes of $M$. sermuletus and M. barbatus are represented in tables (11\&12). These counts were helpful in studying inter-specific variations between species considered. No sexual dimorphism was revealed in the meristic characters of the aforementioned species.

Table 1:- The relationship between some morphometric measurements and standard length of Mullus sermuletus from Alexandria, Egypt for future prediction of missing measurements

\begin{tabular}{|l|l|l|c|}
\hline The equation & \multicolumn{1}{|c|}{ R } & \multicolumn{1}{|c|}{ The equation } & $0.94^{*}$ \\
\hline $\mathrm{FL}=-1.37+1.13 \mathrm{SL}$ & $0.99^{*}$ & $\mathrm{BD}=-1.13+0.26 \mathrm{SL}$ & $0.96^{*}$ \\
\hline $\mathrm{PrD} 1 \mathrm{~L}=4.47+0.30 \mathrm{SL}$ & $0.97^{*}$ & $\mathrm{CPD}=-1.20+0.12 \mathrm{SL}$ & $0.96^{*}$ \\
\hline $\mathrm{PrPL}=4.98+0.26 \mathrm{SL}$ & $0.97^{*}$ & $\mathrm{D} 1 \mathrm{VOFL}=-1.54+0.27 \mathrm{SL}$ & $0.95^{*}$ \\
\hline $\mathrm{PrVL}=2.61+0.30 \mathrm{SL}$ & $0.97^{*}$ & $\mathrm{D} 2 \mathrm{AEFL}=-0.69+0.17 \mathrm{SL}$ & $0.99^{*}$ \\
\hline $\mathrm{PrAL}=1.23+0.65 \mathrm{SL}$ & $0.99^{*}$ & $\mathrm{D} 1 \mathrm{OAEFL}=-5.64+0.52 \mathrm{SL}$ & $0.99^{*}$ \\
\hline $\mathrm{HL}=5.35+0.24 \mathrm{SL}$ & $0.97^{*}$ & $\mathrm{D} 2 \mathrm{EVOFL}=-2.73+0.52 \mathrm{SL}$ & $0.97 *$ \\
\hline $\mathrm{PrOL}=1.88+0.08 \mathrm{SL}$ & $0.86^{*}$ & $\mathrm{D} 2 \mathrm{EvCFL}=-1.62+0.27 \mathrm{SL}$ & $0.95^{*}$ \\
\hline $\mathrm{ED}=2.48+0.06 \mathrm{SL}$ & $0.88^{*}$ & $\mathrm{D} 2 \mathrm{EdCFL}=-0.81+0.24 \mathrm{SL}$ & $0.97 *$ \\
\hline $\mathrm{PtOL}=1.00+0.11 \mathrm{SL}$ & $0.90^{*}$ & AEvCFL$=-0.92+0.24 \mathrm{SL}$ & $0.97 *$ \\
\hline $\mathrm{IOW}=0.64+0.07 \mathrm{SL}$ & $0.93^{*}$ & AEdCFL $=-1.10+0.28 \mathrm{SL}$ & \\
\hline
\end{tabular}

*correlation is significant at the 0.01 level.

Table 2:- The relationship between some morphometric measurements and standard length of Mullus barbatus from Mediterranean Sea Alexandria, Egypt for future prediction of missing measurements

\begin{tabular}{|l|l|l|c|}
\hline The equation & \multicolumn{1}{|c|}{ R } & \multicolumn{1}{|c|}{ The equation } & R \\
\hline FL $=-0.25+1.13 \mathrm{SL}$ & $0.99^{*}$ & $\mathrm{BD}=-2.29+0.26 \mathrm{SL}$ & $0.93^{*}$ \\
\hline $\mathrm{PrD} 1 \mathrm{~L}=2.26+0.30 \mathrm{SL}$ & $0.95^{*}$ & $\mathrm{CPD}=-1.00+0.11 \mathrm{SL}$ & $0.95^{*}$ \\
\hline $\mathrm{PrPL}=2.70+0.26 \mathrm{SL}$ & $0.97^{*}$ & $\mathrm{D} 1 \mathrm{VOFL}=-3.75+0.29 \mathrm{SL}$ & $0.95^{*}$ \\
\hline $\mathrm{PrVL}=2.72+0.28 \mathrm{SL}$ & $0.97^{*}$ & $\mathrm{D} 2 \mathrm{AEFL}=-1.50+0.17 \mathrm{SL}$ & $0.93^{*}$ \\
\hline $\mathrm{PrAL}=0.26+0.64 \mathrm{SL}$ & $0.99^{*}$ & $\mathrm{D} 1 \mathrm{OAEFL}=-4.30+0.51 \mathrm{SL}$ & $0.98^{*}$ \\
\hline $\mathrm{HL}=1.58+0.26 \mathrm{SL}$ & $0.96^{*}$ & $\mathrm{D} 2 \mathrm{EVOFL}=-3.46+0.53 \mathrm{SL}$ & $0.99^{*}$ \\
\hline $\mathrm{PrOL}=-0.48+0.09 \mathrm{SL}$ & $0.85^{*}$ & $\mathrm{D} 2 \mathrm{EvCFL}=1.89+0.24 \mathrm{SL}$ & $0.95^{*}$ \\
\hline $\mathrm{ED}=2.48+0.05 \mathrm{SL}$ & $0.81^{*}$ & $\mathrm{D} 2 \mathrm{EdCFL}=3.55+0.20 \mathrm{SL}$ & $0.93^{*}$ \\
\hline $\mathrm{PtOL}=-0.39+0.12 \mathrm{SL}$ & $0.89^{*}$ & AEvCFL$=1.92+0.22 \mathrm{SL}$ & $0.95^{*}$ \\
\hline $\mathrm{IOW}=0.40+0.06 \mathrm{SL}$ & $0.90^{*}$ & AEdCFL $=-0.47+0.28 \mathrm{SL}$ & $0.97 *$ \\
\hline
\end{tabular}

*correlation is significant at the 0.01 level. 
Table 3:- The basic statistics (Mean \pm standard error and range) of morphometric indices (relative to SL) of males and females of Mullus sermuletus collected from Mediterranean Sea at Alexandria, Egypt.

\begin{tabular}{|l|c|c|c|c|}
\hline \multirow{2}{*}{ Morphometric index } & \multicolumn{2}{|c|}{ Males } & \multicolumn{2}{c|}{ Females } \\
\cline { 2 - 5 } & Mean \pm SE & Range & Mean \pm SE & Range \\
\hline PrD1L & $33.81 \pm 0.14^{* *}$ & $29.60-37.36$ & $33.96 \pm 0.12^{* *}$ & $30.6-36.7$ \\
\hline PrPL & $29.84 \pm 0.14^{* *}$ & $26.15-34.07$ & $30.06 \pm 0.12^{* *}$ & $26.8-33.3$ \\
\hline PrVL & $31.58 \pm 0.12^{* *}$ & $28.5-35.6$ & $31.87 \pm 0.12^{* *}$ & $27.5-34.3$ \\
\hline PrAL & $65.24 \pm 0.14^{*}$ & $62.1-69.2$ & $65.75 \pm 0.14$ & $58.6-69.0$ \\
\hline HL & $28.48 \pm 0.12^{* *}$ & $25.5-32.2$ & $28.53 \pm 0.11^{* *}$ & $25.8-31.4$ \\
\hline PrOL & $9.20 \pm 0.08^{* *}$ & $7.8-11.6$ & $9.45 \pm 0.07^{* *}$ & $7.3-11.2$ \\
\hline ED & $7.64 \pm 0.06^{* *}$ & $6.1-9.0$ & $7.30 \pm 0.05^{* *}$ & $5.8-8.8$ \\
\hline PtOL & $11.64 \pm 0.09^{*}$ & $9.8-14.1$ & $11.78 \pm 0.08$ & $9.0-14.3$ \\
\hline IOW & $7.23 \pm 0.04$ & $6.3-8.3$ & $7.08 \pm 0.04$ & $6.2-8.2$ \\
\hline BD & $25.08 \pm 0.14$ & $21.9-29.6$ & $25.32 \pm 0.15$ & $21.1-28.7$ \\
\hline CPD & $10.65 \pm 0.05^{*}$ & $9.2-11.9$ & $10.97 \pm 0.05^{*}$ & $9.42-12.2$ \\
\hline D1VOFL & $26.00 \pm 0.13$ & $22.8-29.2$ & $26.46 \pm 0.12$ & $23.0-31.9$ \\
\hline D2AEFL & $15.94 \pm 0.09$ & $13.2-17.78$ & $16.14 \pm 0.09$ & $13.3-18.1$ \\
\hline D1OAEFL & $46.90 \pm 0.14^{*}$ & $43.1-50.4$ & $47.35 \pm 0.13^{* *}$ & $43.1-50.9$ \\
\hline D2EVOFL & $50.03 \pm 0.11^{*}$ & $47.8-53.9$ & $50.22 \pm 0.13^{* *}$ & $45.1-54.9$ \\
\hline D2EvCFL & $25.60 \pm 0.09^{*}$ & $23.0-28.2$ & $25.62 \pm 0.11$ & $20.4-29.6$ \\
\hline D2EdCFL & $22.89 \pm 0.11$ & $19.3-25.7$ & $22.85 \pm 0.11$ & $19.3-28.1$ \\
\hline AEvCFL & $23.48 \pm 0.098^{*}$ & $20.6-26.2$ & $23.52 \pm 0.10$ & $20.6-26.7$ \\
\hline AEdCFL & $27.59 \pm 0.12$ & $24.8-31.0$ & $27.56 \pm 0.11^{*}$ & $25.0-33.3$ \\
\hline $\begin{array}{l}\text { Range of correlation } \\
\text { coefficient }\end{array}$ & $(-0.68-0.61)$ & & $(-0.61-0.44)$ \\
\hline N & \multicolumn{2}{|c|}{105} & & 123 \\
\hline
\end{tabular}

** Correlation is significant at the 0.01 level.

* Correlation is significant at the 0.05 level.

Table 4:- The basic statistics (Mean \pm standard error and range) of morphometric indices (relative to HL) of males and females of Mullus sermuletus collected from Mediterranean sea at Alexandria, Egypt.

\begin{tabular}{|l|c|c|c|c|}
\hline \multirow{2}{*}{ Morphometric index } & \multicolumn{2}{|c|}{ Males } & \multicolumn{2}{c|}{ Remales } \\
\cline { 2 - 5 } & Mean \pm SE & Range & Mean \pm SE & $318.8-387.5$ \\
\hline SL & $351.83 \pm 1.51^{* *}$ & $310.3-391.9$ & $351.17 \pm 1.37^{* *}$ & $105.0-130.0$ \\
\hline PrDLL & $118.84 \pm 0.45$ & $107.1-129.7$ & $119.13 \pm 0.40$ & $95.4-117.78$ \\
\hline PrVL & $104.83 \pm 0.33$ & $90.5-113.3$ & $105.42 \pm 0.30$ & $94.1-125.0$ \\
\hline PrAL & $111.00 \pm 0.39^{* *}$ & $102.9-122.2$ & $111.81 \pm 0.42$ & $207.3-257.5$ \\
\hline PrOL & $229.42 \pm 0.81^{* *}$ & $210.3-251.4$ & $230.80 \pm 0.83^{* *}$ & $27.5-40.5$ \\
\hline ED & $32.28 \pm 0.21$ & $27.8-37.5$ & $33.10 \pm 0.20$ & $20.8-29.7$ \\
\hline PtOL & $26.84 \pm 0.18$ & $21.4-30.8$ & $25.60 \pm 0.16^{* *}$ & $33.3-47.9$ \\
\hline IOW & $40.88 \pm 0.26$ & $34.21-47.62$ & $41.31 \pm 0.24^{* *}$ & $20.6-30.0$ \\
\hline BD & $25.44 \pm 0.16^{*}$ & $21.6-31.4$ & $24.86 \pm 0.16$ & $72.7-107.5$ \\
\hline CPD & $88.24 \pm 0.64^{* *}$ & $75.9-108.1$ & $88.91 \pm 0.63^{* *}$ & $73.3-45.0$ \\
\hline D1VOFL & $37.45 \pm 0.24^{* *}$ & $32.0-43.2$ & $38.53 \pm 0.22^{* *}$ & $44.1-66.7$ \\
\hline D2AEFL & $91.45 \pm 0.57^{* *}$ & $78.1-105.4$ & $92.90 \pm 0.54^{* *}$ & $137.5-192.7$ \\
\hline D1OAEFL & $56.07 \pm 0.39^{* *}$ & $48.28-64.86$ & $56.68 \pm 0.38^{* *}$ & $153.1-205.0$ \\
\hline D2EVOFL & $165.09 \pm 1.01^{* *}$ & $137.9-190.5$ & $166.37 \pm 0.93^{* *}$ & $66.7-105.0$ \\
\hline D2EvCFL & $176.03 \pm 0.89^{* *}$ & $148.3-202.4$ & $176.40 \pm 0.89^{* *}$ & $68.4-102.4$ \\
\hline D2EdCFL & $90.09 \pm 0.56^{* *}$ & $74.2-102.7$ & $90.01 \pm 0.56^{* *}$ & $66.7-100.0$ \\
\hline AEvCFL & $80.55 \pm 0.55^{* *}$ & $65.5-92.7$ & $80.27 \pm 0.53^{* *}$ & $84.0-118.2$ \\
\hline AEdCFL & $82.61 \pm 0.53^{* *}$ & $69.0-97.1$ & $82.61 \pm 0.50^{* *}$ & $(-0.32-0.46)$ \\
\hline Range of correlation \\
coefficient
\end{tabular}

** Correlation is significant at the 0.01 level.

* Correlation is significant at the 0.05 level. 
Table 5:- The basic statistics (Mean \pm standard error and range) of morphometric indices (relative to SL) of males and females of Mullus barbatus collected from Alexandria, Egypt.

\begin{tabular}{|l|c|c|c|c|}
\hline \multirow{2}{*}{ Morphometric index } & \multicolumn{2}{|c|}{ Males } & \multicolumn{2}{c|}{ Females } \\
\cline { 2 - 5 } & Mean \pm SE & Range & Mean \pm SE & $26.5-35.6$ \\
\hline PrD1L & $32.31 \pm 0.14^{* *}$ & $28.4-35.0$ & $32.33 \pm 0.13^{*}$ & $26.1-31.8$ \\
\hline PrPL & $28.55 \pm 0.09$ & $26.0-30.0$ & $28.6 \pm 10.09^{* *}$ & $28.0-33.1$ \\
\hline PrVL & $30.47 \pm 0.09^{* *}$ & $27.8-33.3$ & $30.41 \pm 0.10^{* *}$ & $61.2-68.4$ \\
\hline PrAL & $64.24 \pm 0.13$ & $61.5-67.8$ & $64.68 \pm 0.12$ & $25.0-29.6$ \\
\hline Hl & $26.96 \pm 0.10$ & $23.5-28.9$ & $26.92 \pm 0.09^{* *}$ & $6.8-9.5$ \\
\hline PrOL & $7.92 \pm 0.07$ & $5.8-9.5$ & $8.25 \pm 0.06$ & $5.7-8.4$ \\
\hline ED & $7.50 \pm 0.06^{* *}$ & $6.1-9.0$ & $7.09 \pm 0.05^{* *}$ & $9.2-13.0$ \\
\hline PtOL & $11.53 \pm 0.08$ & $9.0-13.4$ & $11.57 \pm 0.08$ & $5.9-7.8$ \\
\hline IOW & $6.65 \pm 0.04$ & $5.7-7.8$ & $6.74 \pm 0.04 *$ & $20.8-29.0$ \\
\hline BD & $23.75 \pm 0.12$ & $21.6-26.9$ & $24.63 \pm 0.14$ & $8.9-11.3$ \\
\hline CPD & $9.83 \pm 0.04$ & $8.9-11.3$ & $10.13 \pm 0.05$ & $22.1-29.3$ \\
\hline D1VOFL & $24.95 \pm 0.12^{*}$ & $22.0-28.5$ & $25.90 \pm 0.13$ & $13.3-18.0$ \\
\hline D2AEFL & $15.54 \pm 0.08$ & $13.3-17.8$ & $16.02 \pm 0.09$ & $44.3-50.3$ \\
\hline D1OAEFL & $46.28 \pm 0.13^{* *}$ & $43.5-49.6$ & $47.33 \pm 0.12^{*}$ & $46.7-53.8$ \\
\hline D2EVOFL & $49.60 \pm 0.11$ & $46.6-52.4$ & $50.34 \pm 0.12^{*}$ & $22.8-28.2$ \\
\hline D2EvCFL & $25.43 \pm 0.11^{*}$ & $23.4-27.6$ & $25.75 \pm 0.11^{* *}$ & $19.5-27.0$ \\
\hline D2EdCFL & $23.08 \pm 0.10^{*}$ & $20.4-25.5$ & $23.00 \pm 0.12^{* *}$ & $21.1-26.5$ \\
\hline AEvCFL & $23.95 \pm 0.09^{* *}$ & $21.9-26.5$ & $24.04 \pm 0.10^{*}$ & $26.1-31.3$ \\
\hline AEdCFL & $27.86 \pm 0.09$ & $25.5-30.5$ & $28.06 \pm 0.09$ & $(-0.50-0.24)$ \\
\hline Range of correlation \\
coefficient & \multicolumn{2}{|c|}{$(-0.29-0.31)$} & & 107 \\
\hline N & & & & \\
\hline
\end{tabular}

** Correlation is significant at the 0.01 level.

* Correlation is significant at the 0.05 level.

Table 6: The basic statistics (Mean \pm standard error and range) of morphometric indices (relative to HL) of males and females of Mullus barbatus collected from Alexandria, Egypt.

\begin{tabular}{|c|c|c|c|c|}
\hline \multirow[t]{2}{*}{ Morphometric index } & \multicolumn{2}{|c|}{ Males } & \multicolumn{2}{|c|}{ Females } \\
\hline & Mean \pm SE & Range & Mean \pm SE & Range \\
\hline $\mathrm{SL}$ & $371.50 \pm 1.44 * *$ & $346.2-426.1$ & $371.89 \pm 1.21$ & $97.2-130.0$ \\
\hline PrD1L & $119.92 \pm 0.43 * *$ & 102.9-129.6 & $120.18 \pm 0.52$ & $97.2-130.0$ \\
\hline PrPL & $105.97 \pm 0.29 * *$ & $100.0-117.4$ & $106.34 \pm 0.30^{* *}$ & $100.0-116.7$ \\
\hline PrVL & $113.15 \pm 0.39 * *$ & $106.7-124.0$ & $113.03 \pm 0.40^{*}$ & $105.7-122.6$ \\
\hline PrAL & $238.54 \pm 0.80 * *$ & $225.0-273.9$ & $240.47 \pm 0.75$ & $223.1-259.3$ \\
\hline PrOL & $29.35 \pm 0.22 * *$ & $23.1-34.3$ & $30.64 \pm 0.19$ & $25.0-37.0$ \\
\hline ED & $27.86 \pm 0.24 * *$ & $23.1-36.0$ & $26.35 \pm 0.17 * *$ & $21.2-32.1$ \\
\hline PtOL & $42.77 \pm 0.25$ & $35.7-46.7$ & $42.98 \pm 0.25 * *$ & $35.5-48.5$ \\
\hline IOW & $24.72 \pm 0.22 * *$ & $21.2-30.8$ & $25.04 \pm 0.17 *$ & $21.9-30.0$ \\
\hline $\mathrm{BD}$ & $88.25 \pm 0.56^{*}$ & $75.0-104.2$ & $91.59 \pm 0.63$ & $76.7-107.7$ \\
\hline $\mathrm{CPD}$ & $36.52 \pm 0.24$ & $32.14-45.8$ & $37.68 \pm 0.22$ & $30.8-42.31$ \\
\hline D1VOFL & $92.65 \pm 0.47$ & $83.3-108.7$ & $96.29 \pm 0.52 *$ & $83.3-108.1$ \\
\hline D2AEFL & $57.72 \pm 0.34$ & $50.0-66.7$ & $59.59 \pm 0.39$ & $50.0-67.7$ \\
\hline D1OAEFL & $171.96 \pm 0.90$ & $155.2-200.0$ & $176.03 \pm 0.78$ & $153.9-194.9$ \\
\hline D2EVOFL & $184.30 \pm 0.87 *$ & $167.9-217.4$ & $187.22 \pm 0.76^{*}$ & $168.0-206.7$ \\
\hline D2EvCFL & $94.51 \pm 0.61 * *$ & $82.1-117.4$ & $95.78 \pm 0.55^{*}$ & $80.8-107.7$ \\
\hline D2EdCFL & $85.76 \pm 0.56 * *$ & 73.3-108.7 & $85.57 \pm 0.56 * *$ & $73.5-103.3$ \\
\hline AEvCFL & $89.00 \pm 0.53 * *$ & 76.9-113.04 & $89.42 \pm 0.52$ & $77.8-100.0$ \\
\hline AEdCFL & $103.52 \pm 0.54 * *$ & $90.0-126.1$ & $104.38 \pm 0.51$ & $88.5-117.7$ \\
\hline $\begin{array}{l}\text { Range of correlation } \\
\text { coefficient }\end{array}$ & \multicolumn{2}{|c|}{$(-0.42-0.33)$} & \multicolumn{2}{|c|}{$(-0.37-0.27)$} \\
\hline $\mathrm{N}$ & \multicolumn{2}{|c|}{100} & \multicolumn{2}{|c|}{107} \\
\hline
\end{tabular}

** Correlation is significant at the 0.01 level.

* Correlation is significant at the 0.05 level. 
Table 7:- The basic statistics (Mean \pm standard error and range) of morphometric indices (relative to SL) of combined sexes of Mullus sermuletus and Mullus barbatus collected from Mediterranean Sea at Alexandria, Egypt.

\begin{tabular}{|c|c|c|c|c|c|}
\hline \multirow{2}{*}{$\begin{array}{l}\text { Morphometric } \\
\text { indices }\end{array}$} & \multicolumn{2}{|c|}{ M. sermuletus } & \multicolumn{2}{|c|}{ M. barbatus } & \multirow[t]{2}{*}{ T-value } \\
\hline & Mean \pm SE & Range & Mean \pm SE & Range & \\
\hline PrD1L & $33.89 \pm 0.09$ & $29.6-37.4$ & $32.32 \pm 0.09$ & $26.5-35.6$ & $13.63 * *$ \\
\hline PrPL & $29.96 \pm 0.09$ & $26.2-34.1$ & $28.58 \pm 0.06$ & $26.0-31.8$ & $13.58 * *$ \\
\hline PrVL & $31.74 \pm 0.09$ & $27.5-35.6$ & $30.44 \pm 0.07$ & $27.8-33.3$ & $12.28 * *$ \\
\hline PrAL & $65.52 \pm 0.10$ & $58.6-69.2$ & $64.46 \pm 0.09$ & $61.2-68.4$ & $7.19 * *$ \\
\hline HL & $28.50 \pm 0.08$ & $24.0-32.2$ & $26.94 \pm 0.07$ & $23.4-29.6$ & $15.69 * *$ \\
\hline PrOL & $9.33 \pm 0.05$ & $7.3-11.6$ & $8.09 \pm 0.05$ & $5.8-9.5$ & $17.36 * *$ \\
\hline ED & $7.45 \pm 0.04$ & $5.8-8.9$ & $7.29 \pm 0.04$ & $5.7-9.0$ & $4.50 * *$ \\
\hline PtOL & $11.70 \pm 0.06$ & $8.2-14.3$ & $11.55 \pm 0.06$ & $9.0-13.4$ & 1.80 \\
\hline IOW & $7.16 \pm 0.03$ & $6.2-9.3$ & $6.70 \pm 0.03$ & $5.7-7.8$ & $11.23 * *$ \\
\hline $\mathrm{BD}$ & $25.21 \pm 0.11$ & $21.1-29.6$ & $24.21 \pm 0.10$ & $20.6-29.0$ & $6.93 * *$ \\
\hline CPD & $10.82 \pm 0.04$ & $9.2-12.17$ & $9.99 \pm 0.04$ & $8.9-11.3$ & $15.05 * *$ \\
\hline D1VOFL & $26.25 \pm 0.09$ & $22.7-31.85$ & $25.44 \pm 0.09$ & $22.0-29.3$ & $6.37 * *$ \\
\hline D2AEFL & $16.05 \pm 0.06$ & $13.2-18.1$ & $15.80 \pm 0.06$ & $13.3-18.0$ & $2.76^{* *}$ \\
\hline D1OAEFL & $47.14 \pm 0.10$ & $43.1-51.0$ & $46.82 \pm 0.10$ & $43.5-50.3$ & 1.78 \\
\hline D2EVOFL & $50.13 \pm 0.09$ & $45.1-54.9$ & $49.99 \pm 0.09$ & $46.6-53.8$ & 0.60 \\
\hline D2EvCFL & $25.61 \pm 0.07$ & $20.4-29.6$ & $25.59 \pm 0.08$ & $22.8-28.2$ & 0.01 \\
\hline D2EdCFL & $22.87 \pm 0.08$ & $19.3-28.1$ & $23.04 \pm 0.08$ & $19.5-27.0$ & -1.35 \\
\hline AEvCFL & $23.50 \pm 0.07$ & $20.6-26.7$ & $24.00 \pm 0.07$ & $21.1-26.5$ & $-5.08 * *$ \\
\hline AEdCFL & $27.58 \pm 0.08$ & $24.8-33.3$ & $27.97 \pm 0.07$ & $25.5-31.3$ & $-4.15 * *$ \\
\hline $\begin{array}{l}\text { Range of correlation } \\
\text { oefficient }\end{array}$ & \multicolumn{2}{|c|}{$(-0.61-0.52)$} & \multicolumn{2}{|c|}{$(-0.46-0.38)$} & \\
\hline $\mathrm{N}$ & \multicolumn{2}{|c|}{228} & \multicolumn{2}{|c|}{207} & \\
\hline
\end{tabular}

* * Significant difference at 0.01 level.

* Significant difference at 0.05 level.

Table 8:- The basic statistics (Mean \pm standard error and range) of morphometric indices (relative to HL) of combined sexes of Mullus sermuletus and Mullus barbatus collected from Mediterranean Sea at Alexandria, Egypt.

\begin{tabular}{|c|c|c|c|c|c|}
\hline Morphometric indices & \multicolumn{2}{|c|}{ M. sermuletus } & \multicolumn{2}{|c|}{ M. barbatus } & \multirow[t]{2}{*}{ T-value } \\
\hline & Mean \pm SE & Range & Mean \pm SE & Range & \\
\hline PrD1L & $351.48 \pm 1.01$ & 310.3-391.9 & $371.70 \pm 0.93$ & $338.5-426.1$ & $-15.54 * *$ \\
\hline PrPL & $119.07 \pm 0.31$ & $105.0-136.4$ & $120.06 \pm 0.34$ & $97.2-130.0$ & $-2.28 *$ \\
\hline PrVL & $105.15 \pm 0.22$ & $90.5-117.8$ & $106.16 \pm 0.21$ & $100.0-117.4$ & $-3.76 * *$ \\
\hline PrAL & $111.50 \pm 0.31$ & $94.1-136.4$ & $113.09 \pm 0.28$ & $105.7-124.0$ & $-4.90 * *$ \\
\hline $\mathrm{HL}$ & $230.31 \pm 0.62$ & $207.3-277.3$ & $239.53 \pm 0.55$ & 223.1-273.9 & $-13.10 * *$ \\
\hline PrOL & $32.74 \pm 0.15$ & $27.5-40.5$ & $30.01 \pm 0.15$ & $23.1-37.0$ & $13.32 * *$ \\
\hline $\mathrm{ED}$ & $26.18 \pm 0.13$ & $20.8-30.8$ & $27.08 \pm 0.15$ & $21.2-36.0$ & $-3.81 * *$ \\
\hline $\mathrm{PtOL}$ & $41.08 \pm 0.18$ & $33.3-47.9$ & $42.88 \pm 0.18$ & $35.5-48.5$ & $-7.78 * *$ \\
\hline IOW & $25.17 \pm 0.12$ & $20.6-35.0$ & $24.89 \pm 0.14$ & $21.2-30.8$ & 1.51 \\
\hline $\mathrm{BD}$ & $88.60 \pm 0.45$ & $72.7-108.1$ & $89.98 \pm 0.43$ & $75.0-107.7$ & $-2.56^{*}$ \\
\hline CPD & $38.03 \pm 0.17$ & $32.0-45.0$ & $37.12 \pm 0.17$ & $30.8-45.8$ & $2.97 * *$ \\
\hline D1VOFL & $92.29 \pm 0.40$ & $76.5-111.4$ & $94.53 \pm 0.37$ & $83.3-108.7$ & $-5.14 * *$ \\
\hline D2AEFL & $56.44 \pm 0.28$ & $44.1-70.5$ & $58.69 \pm 0.27$ & $50.0-67.7$ & $-6.81 * *$ \\
\hline D1OAEFL & $165.9 \pm 0.70$ & $137.5-200.0$ & $174.06 \pm 0.61$ & $153.9-200.0$ & $-10.86 * *$ \\
\hline D2EVOFL & $176.34 \pm 0.65$ & 148.3-211.4 & $185.81 \pm 0.58$ & $167.9-217.4$ & $-12.72 * *$ \\
\hline D2EvCFL & $90.10 \pm 0.40$ & $66.7-109.1$ & $95.16 \pm 0.41$ & $80.8-117.39$ & $-8.93 * *$ \\
\hline D2EdCFL & $80.45 \pm 0.39$ & $65.5-102.4$ & $85.66 \pm 0.40$ & $73.3-108.7$ & $-9.13 * *$ \\
\hline AEvCFL & $82.61 \pm 0.36$ & $66.7-100.0$ & $89.22 \pm 0.37$ & $76.9-113.04$ & $-12.54 * *$ \\
\hline AEdCFL & $96.93 \pm 0.41$ & $82.8-118.9$ & $103.96 \pm 0.37$ & $88.5-126.1$ & $-13.72 * *$ \\
\hline $\begin{array}{c}\begin{array}{c}\text { Range of correlation } \\
\text { coefficient }\end{array} \\
\end{array}$ & \multicolumn{2}{|c|}{$(-0.29-0.53)$} & \multicolumn{2}{|c|}{$(-0.44-0.29)$} & \\
\hline $\mathrm{N}$ & \multicolumn{2}{|c|}{228} & \multicolumn{2}{|c|}{207} & \\
\hline
\end{tabular}

* * Significant difference at 0.01 level.

* Significant difference at 0.05 level. 
Table 9: The allometric coefficients and their standard error $(\mathrm{b} \pm \mathrm{SE})$ of morphometric measurements of males, females and combined sexes of Mullus sermuletus collected from Alexandria, Egypt.

\begin{tabular}{|c|c|c|c|c|c|c|}
\hline \multirow{2}{*}{$\begin{array}{l}\text { Morphometric } \\
\text { measurements }\end{array}$} & \multicolumn{2}{|c|}{ Males } & \multicolumn{2}{|c|}{ Females } & \multicolumn{2}{|c|}{ Combined sexes } \\
\hline & $\mathrm{b} \pm \mathrm{SE}$ & $\mathrm{a}$ & $\mathrm{b} \pm \mathrm{SE}$ & $\mathrm{a}$ & $\mathrm{b} \pm \mathrm{SE}$ & $\mathrm{A}$ \\
\hline PrD1L & $0.86 \pm 0.02-$ & 0.68 & $0.89 \pm 0.02-$ & 0.59 & $0.89 \pm 0.01-$ & 0.58 \\
\hline PrPL & $0.82 \pm 0.02-$ & 0.72 & $0.83 \pm 0.02-$ & 0.69 & $0.84 \pm 0.02-$ & 0.65 \\
\hline PrVL & $0.88 \pm 0.02-$ & 0.55 & $0.91 \pm 0.02-$ & 0.50 & $0.91 \pm 0.02-$ & 0.48 \\
\hline PrAL & $0.95 \pm 0.01 \mathrm{I}$ & 0.85 & $0.97 \pm 0.01 \mathrm{I}$ & 0.78 & $0.97 \pm 0.01 \mathrm{I}$ & 0.76 \\
\hline $\mathrm{HL}$ & $0.80 \pm 0.02-$ & 0.76 & $0.82 \pm 0.02-$ & 0.68 & $0.82 \pm 0.01-$ & 0.68 \\
\hline PrOL & $0.76 \pm 0.05-$ & 0.29 & $0.83 \pm 0.04-$ & 0.22 & $0.84 \pm 0.03-$ & 0.20 \\
\hline ED & $0.79 \pm 0.04-$ & 0.21 & $0.76 \pm 0.03-$ & 0.24 & $0.75 \pm 0.03-$ & 0.25 \\
\hline $\mathrm{PtOL}$ & $0.86 \pm 0.05-$ & 0.23 & $0.91 \pm 0.04-$ & 0.19 & $0.89 \pm 0.03-$ & 0.20 \\
\hline IOW & $0.94 \pm 0.04-$ & 0.10 & $0.94 \pm 0.03 \mathrm{I}$ & 0.09 & $0.93 \pm 0.02 \mathrm{I}$ & 0.10 \\
\hline $\mathrm{BD}$ & $1.07 \pm 0.04+$ & 0.18 & $1.00 \pm 0.04 \mathrm{I}$ & 0.25 & $1.04 \pm 0.02 \mathrm{I}$ & 0.21 \\
\hline CPD & $1.09 \pm 0.03+$ & 0.07 & $1.05 \pm 0.03 \mathrm{I}$ & 0.09 & $1.09 \pm 0.02+$ & 0.07 \\
\hline D1VOFL & $1.05 \pm 0.03 \mathrm{I}$ & 0.20 & $1.02 \pm 0.03 \mathrm{I}$ & 0.24 & $1.04 \pm 0.02 \mathrm{I}$ & 0.21 \\
\hline D2AEFL & $1.03 \pm 0.04 \mathrm{I}$ & 0.13 & $0.99 \pm 0.03 \mathrm{I}$ & 0.17 & $1.02 \pm 0.02 \mathrm{I}$ & 0.15 \\
\hline D1OAEFL & $1.13 \pm 0.02+$ & 0.25 & $1.07 \pm 0.02+$ & 0.33 & $1.10 \pm 0.01+$ & 0.30 \\
\hline D2EVOFL & $1.05 \pm 0.02 \mathrm{I}$ & 0.40 & $1.03 \pm 0.02 \mathrm{I}$ & 0.45 & $1.03 \pm 0.01 \mathrm{I}$ & 0.43 \\
\hline D2EvCFL & $1.05 \pm 0.02 \mathrm{I}$ & 0.20 & $1.04 \pm 0.05 \mathrm{I}$ & 0.21 & $1.04 \pm 0.02 \mathrm{I}$ & 0.21 \\
\hline D2EdCFL & $1.03 \pm 0.03 \mathrm{I}$ & 0.20 & $1.01 \pm 0.03 \mathrm{I}$ & 0.21 & $1.02 \pm 0.02 \mathrm{I}$ & 0.21 \\
\hline AEvCFL & $1.06 \pm 0.03+$ & 0.18 & $1.03 \pm 0.03 \mathrm{I}$ & 0.21 & $1.04 \pm 0.02 \mathrm{I}$ & 0.20 \\
\hline AEdCFL & $1.01 \pm 0.03 \mathrm{I}$ & 0.26 & $1.04 \pm 0.02 \mathrm{I}$ & 0.22 & $1.02 \pm 0.02 \mathrm{I}$ & 0.25 \\
\hline Number & \multicolumn{2}{|c|}{105} & \multicolumn{2}{|c|}{123} & \multicolumn{2}{|c|}{228} \\
\hline
\end{tabular}

$(-)=$ Negative allometric growth, $(+)=$ Positive allometric growth, $(\mathrm{I})=$ Isometric growth . $\mathrm{a}=$ constant of allometric equation.

Table 10:- The allometric coefficients and their standard error $(\mathrm{b} \pm \mathrm{SE})$ of morphometric measurements of males, females and combined sexes of Mullus barbatus collected from Alexandria, Egypt.

\begin{tabular}{|c|c|c|c|c|c|c|}
\hline \multirow{2}{*}{$\begin{array}{l}\text { Morphometric } \\
\text { measurements }\end{array}$} & \multicolumn{2}{|c|}{ Males } & \multicolumn{2}{|c|}{ Females } & \multicolumn{2}{|c|}{ Combined sexes } \\
\hline & $\mathrm{b} \pm \mathrm{SE}$ & $\mathrm{a}$ & $\mathrm{b} \pm \mathrm{SE}$ & $\mathrm{a}$ & $\mathrm{b} \pm \mathrm{SE}$ & $\mathrm{a}$ \\
\hline PrD1L & $0.86 \pm 0.04-$ & 0.62 & $0.91 \pm 0.03-$ & 0.50 & $0.91 \pm 0.02-$ & 0.50 \\
\hline PrPL & $0.94 \pm 0.03-$ & 0.38 & $0.86 \pm 0.02-$ & 0.55 & $0.90 \pm 0.02-$ & 0.46 \\
\hline PrVL & $0.91 \pm 0.03-$ & 0.47 & $0.88 \pm 0.02-$ & 0.55 & $0.89 \pm 0.02-$ & 0.50 \\
\hline PrAL & $0.98 \pm 0.02 \mathrm{I}$ & 0.69 & $0.96 \pm 0.01 \mathrm{I}$ & 0.10 & $0.98 \pm 0.01 \mathrm{I}$ & 0.72 \\
\hline HL & $1.00 \pm 0.04 \mathrm{I}$ & 0.26 & $0.93 \pm 0.02-$ & 0.38 & $0.95 \pm 0.02 \mathrm{I}$ & 0.33 \\
\hline PrOL & $1.10 \pm 0.10+$ & 0.05 & $0.91 \pm 0.05-$ & 0.13 & $1.03 \pm 0.05 \mathrm{I}$ & 0.07 \\
\hline ED & $0.72 \pm 0.07-$ & 0.27 & $0.75 \pm 0.04-$ & 0.23 & $0.70 \pm 0.04-$ & 0.30 \\
\hline PtOL & $1.00 \pm 0.08 \mathrm{I}$ & 0.12 & $1.03 \pm 0.05 \mathrm{I}$ & 0.10 & $1.02 \pm 0.04 \mathrm{I}$ & 0.10 \\
\hline IOW & $0.91 \pm 0.07-$ & 0.10 & $0.84 \pm 0.04-$ & 0.14 & $0.89 \pm 0.03-$ & 0.11 \\
\hline $\mathrm{BD}$ & $0.98 \pm 0.05 \mathrm{I}$ & 0.26 & $1.03 \pm 0.04 \mathrm{I}$ & 0.21 & $1.07 \pm 0.03+$ & 0.17 \\
\hline CPD & $1.09 \pm 0.06+$ & 0.06 & $1.04 \pm 0.03 \mathrm{I}$ & 0.08 & $1.09 \pm 0.03+$ & 0.06 \\
\hline D1VOFL & $1.11 \pm 0.05+$ & 0.15 & $1.06 \pm 0.03+$ & 0.19 & $1.11 \pm 0.03+$ & 0.15 \\
\hline D2AEFL & $0.98 \pm 0.06 \mathrm{I}$ & 0.17 & $1.04 \pm 0.04 \mathrm{I}$ & 0.13 & $1.06 \pm 0.03+$ & 0.12 \\
\hline D1OAEFL & $1.13 \pm 0.03+$ & 0.25 & $1.05 \pm 0.02 \mathrm{I}$ & 0.37 & $1.10 \pm 0.02+$ & 0.30 \\
\hline D2EVOFL & $1.06 \pm 0.02+$ & 0.37 & $1.04 \pm 0.02 \mathrm{I}$ & 0.42 & $1.06 \pm 0.01+$ & 0.38 \\
\hline D2EvCFL & $0.94 \pm 0.05-$ & 0.35 & $0.92 \pm 0.03-$ & 0.38 & $0.95 \pm 0.02 \mathrm{I}$ & 0.32 \\
\hline D2EdCFL & $0.89 \pm 0.05-$ & 0.39 & $0.85 \pm 0.03-$ & 0.48 & $0.88 \pm 0.03-$ & 0.42 \\
\hline AEvCFL & $0.92 \pm 0.04-$ & 0.35 & $0.94 \pm 0.03-$ & 0.32 & $0.95 \pm 0.02 \mathrm{I}$ & 0.31 \\
\hline AEdCFL & $0.99 \pm 0.04 \mathrm{I}$ & 0.30 & $1.01 \pm 0.02 \mathrm{I}$ & 0.27 & $1.01 \pm 0.02 \mathrm{I}$ & 0.26 \\
\hline Number & \multicolumn{2}{|c|}{100} & \multicolumn{2}{|c|}{107} & \multicolumn{2}{|c|}{207} \\
\hline
\end{tabular}

$(-)=$ Negative allometric growth,$(+)=$ Positive allometric growth, $(\mathrm{I})=$ Isometric growth .

$\mathrm{a}=$ constant of allometric equation. 
Table 11:- The pectoral fin rays counts of males and females and combined sexes of Mullus sermuletus and Mullus barbatus from Mediterranean Sea at Alexandria, Egypt

\begin{tabular}{|c|c|c|c|c|c|c|c|c|}
\hline Species & Counts & $\mathrm{N}$ & 15 & 16 & 17 & 18 & Mean \pm SE & T-Value \\
\hline \multirow[t]{3}{*}{ M. sermuletus } & Male & 105 & 2 & 54 & 46 & 3 & $16.47 \pm 0.06$ & \multirow[t]{2}{*}{0.75} \\
\hline & Female & 123 & - & 72 & 49 & 2 & $16.43 \pm 0.05$ & \\
\hline & Comb.sex & 228 & 2 & 109 & 113 & 4 & $16.52 \pm 0.04$ & \multirow[t]{2}{*}{ 3.36* } \\
\hline \multirow[t]{3}{*}{ M. barbatus } & Comb.sex & 207 & - & 136 & 70 & 1 & $16.35 \pm 0.03$ & \\
\hline & Male & 100 & - & 64 & 35 & 1 & $16.37 \pm 0.05$ & \multirow[t]{2}{*}{0.62} \\
\hline & female & 107 & - & 72 & 35 & - & $16.33 \pm 0.05$ & \\
\hline
\end{tabular}

*Significant difference at 0.05 level.

Table 12:- The upper, lower and total gill raker counts of males, females and combined sexes of Mullus sermuletus and Mullus barbatus collected from Mediterranean Sea at Alexandria, Egypt.

\begin{tabular}{|c|c|c|c|c|c|c|c|c|c|c|}
\hline \multirow[t]{2}{*}{ Species } & \multicolumn{10}{|c|}{ Upper gill rakers (UGR) } \\
\hline & counts & $\mathrm{N}$ & 5 & 6 & 7 & 8 & - & - & mean \pm SE & T-value \\
\hline \multirow{3}{*}{$\begin{array}{c}\text { Mullus } \\
\text { sermuletus }\end{array}$} & Males & 105 & 3 & 46 & 51 & 5 & - & - & $6.55 \pm 0.06$ & \multirow[t]{2}{*}{-1.06} \\
\hline & Females & 123 & - & 50 & 69 & 4 & - & - & $6.63 \pm 0.05$ & \\
\hline & Comb. sex & 228 & 3 & 96 & 120 & 9 & - & - & $6.59 \pm 0.04$ & \multirow[t]{2}{*}{$4.34 *$} \\
\hline \multirow{5}{*}{$\begin{array}{c}\text { Mullus } \\
\text { barbatus }\end{array}$} & Comb. sex & 207 & 3 & 126 & 78 & - & - & - & $6.36 \pm 0.04$ & \\
\hline & Males & 100 & - & 59 & 41 & - & - & - & $6.41 \pm 0.05$ & \multirow[t]{2}{*}{1.18} \\
\hline & Females & 107 & 3 & 67 & 37 & - & - & - & $6.32 \pm 0.05$ & \\
\hline & \multicolumn{10}{|c|}{ Lower gill rakers(LGR) } \\
\hline & counts & $\mathrm{N}$ & 16 & 17 & 18 & 19 & - & - & mean \pm SE & T-value \\
\hline \multirow{3}{*}{$\begin{array}{c}\text { Mullus } \\
\text { sermuletus }\end{array}$} & Males & 105 & 3 & 46 & 52 & 4 & - & - & $17.54 \pm 0.06$ & \multirow[t]{2}{*}{-1.01} \\
\hline & Females & 123 & - & 50 & 69 & 4 & - & - & $17.63 \pm 0.05$ & \\
\hline & Comb. sex & 228 & 3 & 96 & 121 & 8 & - & - & $17.59 \pm 0.04$ & \multirow[t]{2}{*}{ 3.53* } \\
\hline \multirow{5}{*}{$\begin{array}{c}\text { Mullus } \\
\text { barbatus }\end{array}$} & Comb. sex & 207 & 2 & 123 & 80 & 2 & - & - & $17.40 \pm 0.04$ & \\
\hline & Males & 100 & 1 & 55 & 43 & 1 & - & - & $17.44 \pm 0.05$ & \multirow[t]{2}{*}{1.13} \\
\hline & Females & 107 & 1 & 68 & 37 & 1 & - & - & $17.36 \pm 0.05$ & \\
\hline & \multicolumn{10}{|c|}{ Total gill rakers(TGR) } \\
\hline & counts & $\mathrm{N}$ & 21 & 22 & 23 & 24 & 25 & 26 & mean \pm SE & T-value \\
\hline \multirow{3}{*}{$\begin{array}{c}\text { Mullus } \\
\text { sermuletus }\end{array}$} & Males & 105 & 1 & 3 & 40 & 8 & 47 & 6 & $24.10 \pm 0.11$ & \multirow[t]{2}{*}{-1.15} \\
\hline & Females & 123 & - & - & 42 & 13 & 63 & 5 & $24.25 \pm 0.09$ & \\
\hline & Comb. sex & 228 & 1 & 3 & 82 & 21 & 110 & 11 & $24.18 \pm 0.07$ & \multirow[t]{2}{*}{ 4.32* } \\
\hline \multirow{3}{*}{$\begin{array}{c}\text { Mullus } \\
\text { barbatus }\end{array}$} & Comb. sex & 207 & - & 5 & 113 & 18 & 69 & 2 & $23.76 \pm 0.07$ & \\
\hline & Males & 100 & - & 1 & 53 & 7 & 38 & 1 & $23.85 \pm 0.10$ & \multirow[t]{2}{*}{1.25} \\
\hline & Females & 107 & - & 4 & 60 & 11 & 31 & 1 & $23.67 \pm 0.09$ & \\
\hline
\end{tabular}

*significant difference at 0.05 level.

\section{Discussion:-}

Morphometric indices of fishes were found to be of taxonomic importance in sex, race, and species identification by many investigators (e.g. Ezzat et al., 1979; Khalil et al., 1983, 1984; Mahmoud, 1988, 1991, 1993; Mahmoud and Mekkawy, 1991; Mekkawy 1987, 1991, 1994, 1997; Harabawy, 1993,2002; Turan, 2004; Lawson, 2010; Mekkawy and Mahmoud, 1992; Osman, 2000; Mekkawy and Mohammad, 2011; Safi et al., 2014; Jawad, 2015 and Mahmoud et al., 2016a,b). In the present study, it was possible to reveal intra-and inter-specific variations in M.sermuletus and M.barbatus by comparing means of morphometric indices.

The type of allometric coefficient was considered by Gould (1966) to be of taxonomic value. The type of allometry was used to study sexual dimorphism in some fish species comprising Mugil cephalus (Grant and Spain, 1975), Mormyrus kanumme (Mekkawy, 1987), Clarias lazera (Mahmoud, 1988), Labeo horie and Labeo forskalii (Mahmoud, 1991), Oreochromis mossambicus (Olivera and Almada, 1995), Barbus bynni (Mahmoud, 2002), Carangoides Bajad and Caranx melampygus (Mahmoud et al., 2016a) and Acanthopagrus bifasciatus (Mahmoud et al., 2016b). The type of alometry was used to study inter-specific variations of four species of genus Epinephelus (Mekkawy et al., 2002) and three Epinephaline species (Mekkawy and Mohammad, 2011). Moreover, the type of 
allometry was used to study intra-and inter-specific variations in some fish species comprising Bagrus bayad and Bagrus docmac (Mahmoud, 1993). In the present study, it was possible to reveal intra-and inter-specific variations of M.sermuletus and M.barbatus according to the type of allometry of the morphometric measurements considered.

Meristic characters were used in intra-and/or inter-specific variations of some fish species comprising Osmerus mordaz (Copeman, 1977), three Synodontis species (Mahmoud and Mekkawy, 1991), Labeo horie and Labeo forskalii (Mahmoud, 1991), Alestis nurse (Mekkawy, 1991), four Tilapiine species (Mekkawy, 1995), four species of genus Epinephelus (Mekkawy et al., 2002), three Epinephaline species (Mekkawy and Mohammad, 2011), Trachurus mediterraneus (Turan, 2004), Carangoides Bajad and Caranx melampygus (Mahmoud et al., 2016a) and Acanthopagrus bifasciatus (Mahmoud et al. 2016b). In the present investigation, the meristic characters of the two species studied showed significant differences in number of pectoral fin rays and upper, lower and total gill rakers counts between M.sermuletus and M.barbatus. No sexual dimorphism was revealed in the meristic characters of the two species studied.

\section{Conclusion:-}

In the present investigation, intra-and inter-specific variations of $M$. sermuletus and M.barbatus were possible in terms of morphometric indices and allometric coefficients. Discrimination between M.sermuletus and M.barbatus was revealed by using pectoral fin rays and upper, lower and total gill rakers counts. No sexual dimorphism was displayed in the meristic characters of the two species studied.

\section{References:-}

1. Abbaspour, R., Rahbar, M. and Karimi, J.M. (2013). Comparative Survey of Morphometrics and Meristics of male and female Anjak fish (Schizocypris brucei, Annandale and Hora, 1920) of Hamoun Wetland in South East Iran, Middle-East Journal of Scientific Research. 14(5): 620-623.

2. Basmidi, A.A.M. (2004). Studies on the population dynamics of some species of genus Lutjanus (family: Lutjanidae) from the Red Sea, Egypt.Ph.D., Thesis, Assiut University, Egypt.

3. Copeman, D.G. (1977). Population differences in rainbow smelt, Osmerus mordax: Multivariate analysis of mensural and meristic data. J. Fish. Res. Board Can. 34: 1220-1229.

4. Costa, J.L., Almeida, P.R. and Costa M.J. (2003). A morphometric and meristic investigation of Lusitanian toadfish Halobatrachus didactylus (Bloch and Schneider, 1980): evidence of population fragmentation on Portuguese coast. J. Scientia Marina. 67(2): 219-231.

5. Deepti, V.A.I., Shrikanya, K.V.L. and Sujatha, K. (2013). Morphometric studies in Hind Grouper Species of Visakhapatnam, central Eastern Coastof India. International Journal of Science and Research. 4(3): $2319-7064$.

6. Elamin, S.M., Ambak, M.A., Samoilys, M.A. and Hamza, M.E. (2011). Some morphometric relationships of coral trouts Plectropomus pessuliferus and Plectropomus areolatus inhabiting Sudanese Red Sea. Advances in Environmental biology J. 5(9): 2860-2865.

7. Golani, D. (1994). Niche separation between colonizing and indigenous goat fish (Mullidae) along the Mediterranean coast of Israel. J. Fish. Biol. 45: 503-513.

8. Gould, S.J. (1966). Allometry and size in ontogeny and phylogeny. Biol.Rev. 41: 587-640.

9. Grant, C.J. and Spain, A.V. (1975). Reproduction, growth and size allometry of Mugil Cephalus Linnaeus (Pisces: Mugillidae) from North Queensland inshore waters. Aust. J. Zool. 23: 181-201.

10. Harabawy, A.S.A. (2002). Biological and Taxonomic Studies on Some Fish Species of the Genus Lethrinus (family: Lethrinidae) from the Red Sea, Egypt and the genus Abramis (family: Cyprinidae) from the Baltic drainage. Ph. D. Thesis, Assiut University, Egypt.

11. Harabawy, A.S.A. (1993). Biological, Biometrics and Electrophoretic Studies on two Bagarid fishes Bagrus bayad (Forsskål, 1775) and Bagrus docmac (Forsskål, 1775) from the Nile at Assiut, Egypt. M. Sc. Thesis, Assiut University Egypt.

12. Huxley, J.S. (1932). Problems of relative growth. Methuen, Co. LTD. London. 276 P.

13. Jawad, L.A. (2015). Study of the vertebral column of the onion trevally, Carangoides caeruleopinnatus (Teleostei: Carangidae) collected from the Red Sea of Oman. Italian Journal of Zoology. 1-7.

14. Khalil, A., Yoakim, E.G. and Mekkawy, I.A.A. (1983). Biometric studies on the Nile charcoid fish, Alestes baremose from Assiut. Assiut Vet. Med. J. 11: 53-58.

15. Khalil, A., Yoakim, E.G. and Mahmoud, U.M. (1984). Biometric and meristic studies on the Nile cyprinoid fish, Labeo niloticus, from Lake Nasser. Assiut Vet. Med. J. 12 (24): 71-78.

16. Lawson, E.O. (2010). Morphometric measurements and meristic count in mudskipper (periophthalmus papilio) 
from mangrove swamps of Lagos lagoon, Nigeria. J. Applied Bioscience. 34: 2166-2172.

17. Mahmoud, U.M., Mehanna, S.F. and Mohammad, A.S. (2016). Sexual dimorphism of Morphometrics and Meristics of Carangoides bajad (Forsskål, 1775) and Caranx melapygus (Cuvier, 1833) from Southern Red Sea, Egypt. International Journal of Science and Research. 5(1): 448-456.

18. Mahmoud, U.M. (1991). Bivariate and multivariate size allometry and sexual dimorphism of Labeo horie and Labeo forskalii from the Nile at Assiut, Egypt. Bull. Fac. Sci., Egypt. 20 (2-E): 39-58.

19. Mahmoud, U.M. and Mekkawy, I.A.A. (1991). Studies on certain meristic characters of three Synodontis species from the Nile at Egypt. Bull. Facl. Sci., Assiut Univ. 20: 1-11.

20. Mahmoud, U.M., El-Gamal, F.I., Mehanna, S.F. and El-Mahdy, S.M, (2016). Study on Morphometric and Meristic Characters of Acanthopagrusbifasciatus (Forsskål, 1775) from Southern Red Sea, Egypt. International Journal of Science and Research. 5(1): 1735-1739.

21. Mahmoud, U.M. (1993). On morphomerics and allometry of Bagrus bayad (Forskal, 1775) and Bagrus docmac (Forskal, 1775) from the Nile at Assiut, Egypt. Vet. Med. J. 28(56): 44-70.

22. Mahmoud, U.M. (1988). Taxonomic studies on some Nile Siluriform fishes. Ph. D. dissertation, Assiut University, Egypt.

23. Mahmoud, U.M. (2002). Sexual dimorphism of morphometrics of Barbus bynni (Foriskal, 1775) from the Nile at Assiut, Egypt. Bull. Fac. Sci. Assiut Univ. 20(1-e): 1-11.

24. Masood, Z., Yasmeen, R.,Rehaman, F., Haider, M.S., Zehra, L., Hassain, M. Y., Rehaman, H., Asim, U., Ahmed, W. and Shah, Q.U. (2015). Comparative studies on some morphometric and meristic characteristics of the scales in four Mugilid species of the family Mugilidae for identifying their significance in taxonomy. Biological Forum- An international Journal. 7(1):176-184.

25. Mazlan, A.G., Chung, Y.S., Zaidi, C.C., Samat, A., Arshad, A., Seah, Y.G., Alam, G.M.and Simon, K.D. (2012). Meristics, Morphometrics and Length-weight relationship of Tropical Silverside, Atherinomorus duodeimalis (Valenciennes in Cuvier and Valenciennes, 1835) in Seagrass and Mangroves Habitats of Tinggi Island, Johor, Malaysia. Asian Journal of Animal and Veterinary Advances. 7 (10): 921-927.

26. Mekkawy, I.A.A. and Mohammad, A.,S. (2011). Morphometrics and meristics of the three epinepheline species: Cephalopholis argus (Bloch and Schneider, 1801), Cephalopholis miniata (Forsskal, 1775) and Variola louti (Forsskal, 1775) from the red sea, Egypt. J. Biol. Sci. 11: 10-21.

27. Mekkawy, I.A.A. (1987). Taxonomic Studies on some Nile Mormyriform fishes. Ph.D. Thesis, Assiut University, Egypt.

28. Mekkawy, I.A.A. and Mahmoud, U.M. (1992). Morphometric and meristic studies of four Labeo species from the Nile at Egypt. J. Egypt Ger. Soc. Zool. 7: 485-513.

29. Mekkawy, I.A.A., Saber, S.A., Shehata, S.M.A. and Osman, A.G.M. (2002). Morphometrics and meristics of four species of genus Epinephelus (Family Serranidae) from the Red Sea, Egypt. Bull. Fac.Sci.Assiut. Univ. 31: 21-41.

30. Mekkawy, I.A.A. (1997). Meristic and morphometric patterns of three Egyptian Bagrus species. J. Egypt. Ger. Soc. Zool. 22: 93-121.

31. Mekkawy, I.A.A. (1994). Description of $O$. ismailiaensis sp. n., and its hybrid with O.niloticus (Linnaleus, 1758) Perciformes, Cichlidae from Egypt. Bull. Fac. Sci., Assiut University. 23(2E): 1-27.

32. Mekkawy, I.A.A, (1995). Intra-and inter-specific variations in the meristic characteristics of some tilapiine species of Egypt. Assiut Vet. Med. J. 33: 11-45.

33. Mekkawy, I.A.A. (1991). Multivariate analysis of the morphometric and meristic characters of the Nile charcoid fish, Alestis nurse (Ruppel, 1832) from the Nile at Egypt. Assiut Vet. Med. J. 26: 35-52.

34. Nelson, J.S. (1984). Fishes of the World. In: John Wiley and Sons.New York.

35. Obady, Y.H. (2003). Biological and taxonomic studies on some species of Genus Siganus (Family: Siganidae) from the Red Sea Egypt. M.Sc., Thesis, Assiut Univ., Egypt.

36. Oliveira, R.F. and Almada, V.C. (1995). Sexual dimorphism and allometry of external morphology in Oreochromis mossambicus. J. Fish. Biol. 46:1055-1064.

37. Osman, A.G.M. (2000). Taxonomical and biological studies of some species of genus Epinephelus (Family: Serranidae) from the Red Sea, Egypt. M. Sc. Thesis, Al-Azhar University, Cairo.

38. Randall, J.E. and King, D.R. (2009). Parupeneus fraserorum, a new species of goatfish (Perciformes: Mullidae) from South Africa and Madagascar. Smithiana Bull.10: 31-35.

39. Randall, J.E. and Heemstra, P.E. (2009). Three new goatfishes of the genus Parupeneus from the western Indian Ocean, with resurrection of P. seychellensis. Smithiania. Pub. Aquatic Biodiversity. 5: 37-49.

40. Safi, A., Khan, M.A. and Khan, M.Z. (2014). Study of some morphometric and meristic characters of striped piggy fish, Pomadasyss tridens(Forsskal, 1775) from Karachi Coast, Pakistan.The Journal of 
Zoology Studies. 1(4): 1-6.

41. Sajina, M., Chakraborty, S.K., Jaiswar, A.K. and Sudheesan, D., (2013). Morphometric and meristic analyses of Horse Mackerel, Megalaspis cordyla (Linnaeus, 1758) Populations along the Indian Coast. Indian J. Fish. 60(4): 27-34.

42. Simon, K.D., Bakar, Y., Temple, S.E. and Mazlan, A.G. (2010). Morphometric and meristic variation in two congeneric archer fishes Toxotes chatareus inhabiting Malaysia coastal waters. J. Zhejiang Univ-Sci. B (Biomed \& Biotechnol). 11(11): 871-879.

43. Smith, P.J. and Paulin, C.D. (2003). Genetic and morphological evidence for a single species of pink ling (Genypterus blacodes) in New Zealand waters. New Zealand Jornal of Marine and Freshwater Research. 37(1): 183-194.

44. Turan, C. (2004). Stock identification of Mediterranean horse mackerel (Tachurus mediterraneus) using morphometric and meristic characters. ICES J. Mar. Sci. 61: 774-781.

45. Uiblein, F. and Heemstra, P.C. (2011). A new goatfish, Upeneus seychellensis n. sp. (Mullidae), from the Seychelles Bank, with remarks on Upeneus guttatus and a key to Western Indian Ocean Upeneus species. Marine Biology Research. 7: 637-650.

46. Uiblein, F. and Heemstra, P.C. (2010). A taxonomic review of the Western Indian goatfishes of the genus Upeneus (Family Mullidae) with descriptions of four new species. Smithiana Bull. 11: 35-71.

47. Whitehead, P.J.P., Bauchot. M.L., Hureau, J.C., Nielsen, J. and Tortonese, E. (1986). Fishes of the northeastern Atlantic and the Mediterranean. Vol. 2-3: 511-1473.

48. Zubia, M., Rehana, Y.F., Katselis, G., Omer, M.T., Zehra, L., Hossain, Y.M. and Samee, H.M., (2015). Comparative Survey of Morphometric and Meristic studies of four mullet species of family Mugillidae from Pakistan in relation to total body length. Indian Journal of Geo-Marine Science. 44(4): 11p. 